\author{
Psychophysiological Responses to Auditory Change \\ Lorraine Chuen, David Sears and Stephen McAdams \\ McGill University
}

\begin{abstract}
Author Note
Lorraine Chuen, Department of Psychology, McGill University.

David Sears and Stephen McAdams, Department of Music Research, Schulich School of
\end{abstract} Music, McGill University.

This is the peer reviewed version of the following article: Chuen, L., Sears, D., \& McAdams, S. (2016). Psychophysiological responses to auditory change. Psychophysiology, which has been published in final form at doi: 10.1111/psyp.12633. This article may be used for non-commercial purposes in accordance with Wiley Terms and Conditions for Self-Archiving

The authors wish to thank Bennett K. Smith for designing the experimental interface and Finn Upham and Meghan Goodchild for assistance with analysis of respiratory signals. Portions of this work were presented at the 2013 meeting of the Society for Music Perception and Cognition, Toronto and the 2013 Auditory Perception, Cognition and Action Meeting, Toronto.

This work was supported by grants from the Canadian Natural Sciences and Engineering Research Council (RGPIN 2015-05280) and the Canada Research Chairs program (\#950223484) award to Stephen McAdams.

Correspondence concerning this article should be addressed to Stephen McAdams, Schulich School of Music, McGill University, 555 Sherbrooke St. W., Montreal, QC, Canada H3A 1E3.

Contact: stephen.mcadams@mcgill.ca 


\title{
PSYCHOPHYSIOLOGICAL RESPONSES TO AUDITORY CHANGE
}

\begin{abstract}
A comprehensive characterization of autonomic and somatic responding within the auditory domain is currently lacking. We studied whether simple types of auditory change that occur frequently during music listening could elicit measurable changes in heart rate, skin conductance, respiration rate and facial motor activity. Participants heard a rhythmically isochronous sequence consisting of a repeated standard tone, followed by a repeated target tone that changed in pitch, timbre, duration, intensity or tempo, or that deviated momentarily from rhythmic isochrony. Changes in all parameters produced increases in heart rate. SCR magnitude was affected by changes in timbre, intensity, and tempo. Respiratory rate was sensitive to deviations from isochrony. Our findings suggest that music researchers interpreting physiological responses as emotional indices should consider acoustic factors that may influence physiology in the absence of induced emotions.
\end{abstract}

Keywords: auditory change, facial EMG, heart rate, skin conductance, respiration rate 


\section{PSYCHOPHYSIOLOGICAL RESPONSES TO AUDITORY CHANGE}

The ability to detect changes in our auditory environment is an essential component of auditory scene analysis. Research on the orienting response-a complex of physiological and behavioral responses elicited by novel sensory stimulation-indicates that humans are highly sensitive to even small, seemingly innocuous auditory changes (Sokolov, 1963). Functionally, studies have suggested that this reaction to novel stimuli in our environment reflects the reallocation of attention (Siddle, 1991), as well as an increase in stimulus sensitivity (Stekelenburg \& van Boxtel, 2002). Much attention has been devoted to how this response is displayed physiologically, where markers of orienting typically include the skin conductance response (Barry \& Sokolov, 1993) and heart rate deceleration (Graham \& Clifton, 1966), as well as inhibition of respiration and EMG activity in the lower facial muscles (Stekelenburg \& van Boxtel, 2002).

Despite advances made in our understanding of the physiological components of the orienting reflex, only a small body of research has focused on the effects of acoustic parameters on autonomic and somatic responding. This research indicates that the orienting response can be elicited by changes in tone frequency (Siddle \& Heron, 1976) and acoustic intensity (Siddle \& Heron, 1977), with the magnitude of the skin conductance response reflecting the magnitude of the frequency or intensity change. Changes in frequency, intensity, and rise-time of the temporal envelope at the onset of a sound also elicit heart rate responses: An early deceleration in heart rate follows the onset of frequency and intensity change (Lyytinen, Blomberg, \& Näätänen, 1992; Siddle \& Heron, 1976, 1977), and a later heart rate acceleration follows changes in risetime (Lyytinen et al., 1992). Research also indicates that presentation of high-intensity auditory stimuli cause increases in corrugator activity (Dimberg, 1990), although to the best of our 


\section{PSYCHOPHYSIOLOGICAL RESPONSES TO AUDITORY CHANGE}

knowledge, no work has focused on how changes in intensity or other parameters affect somatic activity.

The majority of research on auditory change processing focuses instead on the mismatch negativity $(\mathrm{MMN})$, a component of the auditory event-related potential that is elicited by violations of acoustic regularities (Näätänen \& Winkler, 1999). This research indicates that the MMN amplitude is directly related to the perceptual dissimilarity between the standard and change stimulus (Tiitinen, May, Reinikainen, \& Näätänen, 1994; Toiviainen et al, 1998). Importantly, there are few analogous studies investigating the degree of the auditory change's effect on the magnitude of autonomic and somatic responses. Given that stimulus changes that cause the MMN do not necessarily elicit comparable responses in the autonomic nervous system (Lyytinen et al., 1992), the current study investigates how changes on various acoustic parameters affect autonomic and somatic physiology.

Additionally, little research on the orienting response has been conducted on musically relevant parameters, such as rhythmic regularity, tempo, and timbre - the unique quality of a sound that allows us to distinguish between instruments. A greater understanding of how changes in these musically relevant parameters relate to autonomic and somatic physiology is particularly important given the growing popularity of measuring physiological responses to complex musical stimuli (e.g., Coutinho \& Cangelosi, 2011; Gomez \& Danuser, 2007). To be sure, music presents a challenging stimulus domain in experimental research as a consequence of the numerous individual acoustic features that vary constantly during music listening. The current study's investigation of the effects of acoustic change in specific sound parameters on physiological response will allow music researchers to better contextualize research on the relationship between acoustic features and physiology during music listening. 


\section{PSYCHOPHYSIOLOGICAL RESPONSES TO AUDITORY CHANGE}

The central aim of the current study was to characterize physiological responding to changes in acoustic parameters that are also commonly manipulated in music. These parameters were pitch, timbre, intensity, inter-onset interval (or tempo), tone duration, and rhythmic isochrony. We chose to employ a repetition-change paradigm to measure the orienting reflex, allowing for the controlled study of the effect of these acoustic parameters on physiology. We introduced a standard stimulus, and after a period of repetition, presented a novel stimulus representing varying degrees of auditory change, which was then repeated. Autonomic physiological measures (heart rate, respiration rate, and skin conductance response) and somatic measures (zygomaticus and corrugator muscle activity) that are often employed in music research were collected. Experiment 1 evaluated whether changes in pitch, timbre and rhythmic isochrony could elicit changes in autonomic nervous system activity and facial motor activity. Experiment 2 examined responses to changes in pitch, timbre, loudness, tempo, and tone duration.

Consistent with previous research, we expected to observe evidence of orienting responses to auditory change, which consist of skin conductance responses, decelerations in heart rate, decreases in respiration rate, and a decrease in zygomaticus activity. We also considered the possibility that some auditory changes (e.g., intensity increases) would induce defensive responses, which would be characterized by the presence of skin conductance responses (SCRs) and accelerations in heart rate (Cook \& Turpin, 1997; Graham \& Clifton, 1966), although there is some controversy concerning the ability of the directionality of heart rate change to differentiate orienting and defensive responses (Barry \& Maltzman, 1985).

Overall, we predicted that the degree of deviation between the standard and comparison tones would influence the magnitude of the physiological response. This prediction is in line 


\section{PSYCHOPHYSIOLOGICAL RESPONSES TO AUDITORY CHANGE}

with Sokolov's (1963) concept of a neuronal model of orienting and Gati \& Ben-Shakhar's (1990) feature-matching model of orienting, both of which suggest that the degree of stimulus novelty - and thus, the magnitude of orienting - is inversely proportional to the closeness of the match between the incoming stimulus and the established short-term memory representation (neuronal model) of the past stimulus. This prediction would also agree with evidence from the auditory change detection literature, which indicates that the MMN amplitude is directly related to the perceptual dissimilarity between the standard and change stimuli (Tiitinen et al., 1994; Toiviainen et al., 1998).

In the current experiments, we defined the degree of change in pitch as a difference in log fundamental frequency (pitch height in $\log \mathrm{Hz}$ ) between two notes, loudness as a difference in intensity (decibels or $\mathrm{dB}$ ), tempo as a difference in inter-onset interval (IOI in $\mathrm{ms}$ ), and duration as a difference in tone duration (ms). Timbre change was operationalized as a combination of three dimensions: spectral flux, which represents the degree of variation of the spectral envelope over time (Krimphoff, McAdams, \& Winsberg, 1994); spectral centroid, which corresponds to our experience of auditory "brightness" (Krimphoff et al., 1994); and log rise time, which corresponds to the duration of the attack portion of a tone's amplitude envelope (McAdams, Winsberg, Donnadieu, Krimphoff, \& De Soete, 1995). We also predicted that temporal expectations might shape the listener's physiological response. Listeners are sensitive to the temporal regularities of auditory events, and perturbations from regularity have a number of effects, from influencing our ability to detect tempo deviations (McAuley \& Kidd, 1998) to altering tapping production behavior (Madison \& Merker, 2004). To assess the role of a departure from isochrony on physiological activity, we therefore included an isochrony 


\section{PSYCHOPHYSIOLOGICAL RESPONSES TO AUDITORY CHANGE}

manipulation in Experiment 1, in which the target tone could fall earlier or later than the established IOI.

\section{Experiment 1}

\section{Method}

Participants. Forty participants ( 20 male; $M=23$ years, $S D=4$ years) took part in the experiment. Participants were recruited in Montreal, Canada. All participants underwent a brief hearing exam to ensure that their pure-tone hearing thresholds were within $20 \mathrm{~dB}$ of standard hearing levels at octave-spaced frequencies from $250 \mathrm{~Hz}$ to 8,000 $\mathrm{Hz}$ (ISO 389-8, 2004; Martin \& Champlin, 2000). All participants gave informed consent. The study was certified by the McGill University Review Ethics Board.

Stimuli. Each sequence faded in from silence to $65 \mathrm{~dB}$ SPL across the first four tones and faded out to silence across the last four tones. For all sequences, the reference tone was a synthetic tone with a bassoon-like timbre and a pitch of Eb4 $(311 \mathrm{~Hz}$ fundamental frequency). The auditory stimuli consisted of 144 trials of isochronous sequences of 24 events presented at a tempo of 100 beats per minute (inter-onset interval of $600 \mathrm{~ms}$ ). Each sequence was approximately $15 \mathrm{~s}$ in duration. On the target tone (event $10,11,12,13,14$, or 15), the sequence would contain either a change in pitch, a change in timbre or no change. Tones were drawn from the set used by McAdams et al. (1995) and had a sampling rate of $44.1 \mathrm{kHz}$ and 16-bit amplitude resolution. In the pitch block, all tones were $499 \mathrm{~ms}$ in duration. In the timbre block, the durations of tones were: $499 \mathrm{~ms}$ (standard tone; Bassoon), $512 \mathrm{~ms}$ (Trumpet), $574 \mathrm{~ms}$ (French Horn), and $546 \mathrm{~ms}$ (Obochord; the obochord was conceived as a digital hybrid between an oboe and a harpsichord; Wessel, Bristow, \& Settel, 1987). The sounds had been equalized for loudness and perceived duration by McAdams et al. (1995). 


\section{PSYCHOPHYSIOLOGICAL RESPONSES TO AUDITORY CHANGE}

Variations in pitch. On the target tone, $25 \%$ of the trials consisted of no change in pitch, $25 \%$ contained an upward shift of four semitones to G4 $(392 \mathrm{~Hz}$ fundamental frequency, an interval of a major third), 25\% an upward shift of 6 semitones to B4 (494 Hz, an interval of a tritone), and $25 \%$ an upward shift of 11 semitones to E5 (659 Hz, an interval of a major seventh). Pitch shifting of the original Eb4 bassoon-like tone was performed using AudioSculpt (IRCAMCentre Pompidou, Paris). The authors verified that the pitch shifts resulted in no audible artifacts or changes in loudness.

Variations in timbre. On the target tone, $25 \%$ of the sequences experienced no change in timbre, $25 \%$ contained a shift to a trumpet-like tone, $25 \%$ to a French horn-like tone, and $25 \%$ to an obochord tone. For these stimuli, variations in timbre result from changes along three dimensions: spectral flux, spectral centroid, and log rise time (McAdams et al., 1995). With respect to the (bassoon-like) reference tone, the trumpet timbre varied primarily along the spectral flux dimension, the French horn along the spectral centroid dimension, and the obochord along the log rise time dimension (see Table 1 for relative distances along primary dimensions; McAdams et al., 1995).

\begin{tabular}{lcccc}
\hline Instrument & Attack time & Spectral centroid & Spectral flux & Global distance \\
\hline French horn & 0.9 & $\underline{3.1}$ & 0.5 & 3.8 \\
Trumpet & 0.2 & 0.1 & $\underline{2.4}$ & 3.2 \\
Obochord & $\underline{4.9}$ & 0.5 & 0.7 & 5.2 \\
\hline
\end{tabular}

Table 1. Distances (on a scale of 1-9) from the reference bassoon-like tone along each dimension of the McAdams et al. (1995) space (primary dimension of interest for each timbre is underlined) and the global distance including specificities on each instrument. 


\section{PSYCHOPHYSIOLOGICAL RESPONSES TO AUDITORY CHANGE}

Variations in rhythmic isochrony. One third of the trials in the pitch and timbre blocks were completely isochronous (On condition), in one third the onset of the target tone was advanced by $80 \mathrm{~ms}$ (Early condition), and in one third it was delayed by $80 \mathrm{~ms}$ (Late condition). The inter-onset interval (IOI) of the target tone and the following tone was the standard $600 \mathrm{~ms}$, and all subsequent tones maintained this standard IOI. The non-isochronous tones did not fall on the beat established by the preceding tones and thus were presumed to violate a listener's temporal and rhythmic expectations.

Apparatus. The stimuli were reproduced using an M-Audio Audiophile 192 sound card (Avid, Irwindale, CA), converted to analog using a Grace Design m904 monitor system (Grace Design, Boulder, CO), and presented over a pair of Sennheiser HD 280 Headphones (Sennheiser Electronics, GmBH, Wedemark, Germany). Stimuli were presented at a level of $65 \mathrm{~dB}$ SPL on average as measured with a Bruel \& Kjaer (Holte, Denmark) Type 2250 sound level meter (Aweighting, fast setting) and a Type 4157 artificial ear to which the headphone was coupled. The experimental program, subject interface, and data collection were programmed using the Pure Data signal processing software environment ( $\mathrm{Pd}$ version 0.42.5, http://puredata.info, last accessed September 14, 2015) controlled by the PsiExp software environment (Smith, 1995).

All physiological measurements were recorded with a ProComp Infiniti Monitor (SA7500) with several biosensors (Thought Technology Ltd., Montreal, Canada). Respiration was measured using a respiration monitor (stretch transducer, SA9311M) attached around the chest of the participant just below the pectoral muscles. Blood volume pulse was measured using a photoplethysmograph $(\mathrm{SA} 9308 \mathrm{M})$ on the palmar side of the distal phalange of the middle finger of the non-dominant hand. Skin conductance was measured using electrodes (SA9309M) on the distal phalange of the index and ring fingers of the non-dominant hand. Activation of 


\section{PSYCHOPHYSIOLOGICAL RESPONSES TO AUDITORY CHANGE}

muscles during facial expression was measured using two MyoScan-Pro EMG electrodes (SA9401M-60) placed over and in line with the corrugator supercilii and zygomaticus major muscles, which are active during frowning and smiling, respectively. EMG electrodes were placed on the side of the face contralateral to the dominant hand.

The samples were sent to the computer via a USB interface optically coupled to the ProComp. The USB data were decoded and recorded by a monitor process running on the workstation. The synchronization of data recording by the USB monitor process and signal generation by Pure Data was accomplished by OSC (Open Sound Control, http://opensoundcontrol.org, last accessed September 14, 2015) messages sent by the PsiExp program. Synchronization latency was less than one sample period (4 ms).

Procedure. Biosensors were attached to the face, hands, and around the chest. Participants read the instructions and could ask any questions of the experimenter. Each trial consisted of a 5-s countdown shown with numbers on the screen to prepare the participant to remain still during stimulus presentation. The auditory sequence was then played. As an attentional control task, following each auditory sequence participants were required to indicate whether they heard a change during the sequence by typing " $\mathrm{Y}$ " for yes or " $\mathrm{N}$ " for no on the computer keyboard and then pressing the carriage return. Upon pressing the return key, the countdown for the next trial began. The inter-trial interval therefore consisted of the 5-s countdown, the duration of the 24-tone stimulus sequence, and the participant's response time (roughly $7 \mathrm{~s}$ on average between stimuli).

The main experiment consisted of a total of 144 trials divided into two blocks of 72 auditory sequences each. Each block was approximately $30 \mathrm{~min}$ in duration and consisted entirely of either pitch variations or timbre variations within the auditory sequence. Block order 


\section{PSYCHOPHYSIOLOGICAL RESPONSES TO AUDITORY CHANGE}

was counterbalanced, and trial order within each block was randomized. Participants were given a two-minute break between blocks. To familiarize the participants with the procedure and control task, the experimental session began with two practice trials.

Data Preprocessing. Blood volume pulse, respiration, and electrodermal activity were collected at a sampling rate of $256 \mathrm{~Hz}$ and were then processed offline in MATLAB (The MathWorks, Inc., Natick, MA) to extract relevant features: respiration rate (breaths per minute or $\mathrm{BrPM}$ ), heart rate (beats per minute or $\mathrm{BPM}$ ), and skin conductance response magnitude (microSiemens or $\mu \mathrm{S}$ ). Before sampling the analog signal at $256 \mathrm{~Hz}$, the MyoScan-Pro EMG electrodes performed analog rectification and band-pass filtering $(20-500 \mathrm{~Hz})$, and this signal was then converted to an equivalent of RMS using a first-order IIR low-pass filter with a time constant of $20 \mathrm{~ms}$. So no further processing steps were performed for the corrugator and zygomaticus EMG signals. To remove artifacts and extraneous noise, all other signals were lowpass filtered with a fourth-order, Butterworth zero-phase filter, which avoids phase shifting the signal by convolving the filter impulse response with itself in time reverse. Skin conductance level (SCL) was filtered with a cut-off frequency of $0.3 \mathrm{~Hz}$, blood volume pulse with a cut-off frequency of $2.2 \mathrm{~Hz}$, and respiration with a cut-off frequency of $2 \mathrm{~Hz}$. All filtered signals were visually inspected for remaining artifacts, and trials with abnormal signals within the time window of interest ( -1 to $4 \mathrm{~s}$ after auditory change) were removed from the analysis.

To extract heart rate from the blood volume pulse signal, inter-beat intervals were calculated by locating local maxima and translating the resulting beat period to a beats-perminute measure at that time point (Berntson, Quigley, \& Lozano, 2007). Samples that were less than half or more than two times the median were replaced by the median. In order to create a continuous time series, the resulting times series were then up-sampled back to $256 \mathrm{~Hz}$ using 


\section{PSYCHOPHYSIOLOGICAL RESPONSES TO AUDITORY CHANGE}

linear interpolation. Like the other physiological signals, the continuous heart-rate time series was then binned into 1-second epochs as described below.

A technique involving cross-correlation of the filtered respiratory signal with sinusoidal signals of different frequencies was used to estimate the time-varying frequency of the respiration. To extract instantaneous respiration rate in breaths per minute (BrPM) from the respiration belt signal over fairly small time periods, for which average inter-inhalation interval would be difficult to assess, we adapted a spectral analysis technique from Müller (2007, chap. 3) originally designed for pitch analysis and beat detection on audio signals (Upham, 2014). It identifies the principle frequency of oscillation by the peak correlation between 10-s frames of the signal and a bank of sinusoidal signals of appropriate frequency. The procedure first downsamples the filtered respiration signal to a $10-\mathrm{Hz}$ sampling rate and calculates the first-order difference, thus representing the rate of change in the expansion and contraction of the thorax. It then computes the cross-correlations between the resulting time series and a bank of 101 sinusoids with frequencies of 5-30 BrPM at 0.25 BrPM increments, using 10-s frames and a hop size of 1 sample $(0.1 \mathrm{~s})$. To account for anomalous respiratory behaviors such as yawning, one component of the set of sinusoids was generated at $1 \mathrm{BrPM}$, resulting in a monotonic set of samples over the 10-s window. Within this window, this component would cross-correlate well with breathing rates of $3 \mathrm{BrPM}$ or less. Frames with absolute correlation coefficients greater than 0.5 were sampled for peak values that fit the short segment of the respiration cycle in phase and frequency. From the resulting series, maxima were extracted, interpolated, and smoothed with a 0.1-Hz 4th-order Butterworth low-pass filter. This technique thereby provides a measure of the time-varying breathing rate. This breathing-rate signal was then up-sampled to $256 \mathrm{~Hz}$ for comparison with other series in the data set. This technique provides a more robust estimate of 


\section{PSYCHOPHYSIOLOGICAL RESPONSES TO AUDITORY CHANGE}

time-varying respiration rate on fairly short signals by making use of more signal information than the onset of each breath.

Heart rate, respiration rate, zygomaticus and corrugator signals were averaged offline into 1 -s epochs ranging from $1 \mathrm{~s}$ before to $4 \mathrm{~s}$ following the auditory change. Epochs were calculated by taking the mean of all values falling between $0.5 \mathrm{~s}$ before and after the time point of interest. Using a method similar to that described by Bradley and Lang (2000a), the magnitude of the physiological response was measured by deviating epoch means from the participant's baseline physiological activity in that trial, which was taken as the average signal level $1 \mathrm{~s}$ immediately preceding the onset of the change. Epoch values per participant and per condition were averaged across the six repetitions for subsequent analyses.

To quantify SCR magnitude, the first peak and trough occurring after the onset of auditory change were located in the skin conductance signal, and the response amplitude was taken as the difference between the trough and peak values. Signals without a peak were assigned a value of zero. To calculate SCR magnitude, which includes trials with amplitude values of zero, the amplitude data were normalized using a $\log ($ amplitude +1$)$ transformation, where 1 is added to all SCR amplitudes before calculating the $\log$, as the $\log$ of 0 is undefined (Boucsein, 2012). SCR magnitude per condition and per participant was then averaged across all six repetitions of the condition for subsequent statistical analyses.

Given the short duration of stimulus sequences prior to auditory change, the square root of the mean squared differences between successive inter-beat intervals (RMSSD) was used as an index of heart rate variability. RMSSD was treated as a between-subjects covariate in the analyses that follow, so the RMSSD was calculated for the pre-change portion of each trial and then averaged across all trials in the experimental session for each participant. Finally, to 


\section{PSYCHOPHYSIOLOGICAL RESPONSES TO AUDITORY CHANGE}

consider the effect of the respiratory sinus arrhythmia (RSA) on heart rate, the proportion of inhalations vs. exhalations during the target event for each condition (across 6 repetitions) was calculated per participant.

Analyses. For SCR and proportion of inhalations, a repeated-measures ANOVA was conducted with Auditory Change along a given dimension (timbre, pitch, rhythmic isochrony) as a within-subjects factor.

For all other processed physiological signals (heart rate, respiration rate, zygomaticus and corrugator EMG activity), a mixed-design, repeated-measures ANOVA was conducted to determine the effect of auditory change on the physiological response over time. For each block, Epoch and Auditory change were treated as within-subjects factors. In instances in which sphericity was violated (indicated by Mauchly's test), we report the original degrees of freedom, the value of the Greenhouse-Geisser $\varepsilon$, and adjusted $p$-values. Effect size is reported as generalized $\eta^{2}$ (notated $\eta_{G}^{2}$; Bakeman, 2005; Olejnik \& Algina, 2003), because $\eta_{G}^{2}$ allows for comparison of effect sizes across both between-subjects and within-subjects designs. When posthoc tests are used Bonferroni-Holm-corrected p-values are reported.

We interpreted Epoch $\times$ Auditory Change interactions as an indicator that the auditory change elicited a measurable effect on the physiological signal over time (i.e., the effect of change condition was not due to baseline differences between conditions existing before the target tone). If an interaction existed, we conducted a separate polynomial trend analysis on Epoch to determine the nature of the interaction: a different trend analysis was done at each level of the auditory change factor, and additional pair-wise trend comparisons were conducted to determine whether the change condition trends differed significantly from the no-change condition trend. The trend analysis was conducted with a generalized least squares approach in 


\section{PSYCHOPHYSIOLOGICAL RESPONSES TO AUDITORY CHANGE}

$\mathrm{R}$ by specifying an orthogonal cubic polynomial for Epoch and using a first-order autoregressive specification for correlations among repeated measures. For trend analyses, we report the coefficient estimate, $B$, and the ratio between the estimate and the standard error, $t$. Main effects of Epoch were not reported in the analyses that follow, however, as physiological signals generally change over time; only the Epoch x Auditory Change interaction was of interest.

Post-hoc analyses were conducted on significant main effects to determine significant differences between conditions. For significant Epoch $\times$ Auditory Change interactions, pairwise comparisons were conducted to determine change-condition differences within particular epochs. Similarly, for significant effects of Auditory Change on SCR magnitude, pairwise, one-tailed ttests were conducted to determine which change conditions elicited a significantly greater SCR magnitude than the no-change condition. All $p$-values were adjusted using the Bonferroni-Holm method for all pairwise comparisons. Marginally significant effects are only reported for pairwise comparisons and Parameter $\times$ Epoch interactions (our primary effects of interest).

One participant was excluded from the heart rate analysis due to consistent artifacts present in the blood volume pulse signal. After excluding this participant, on average $3.9 \%$ (SD $=0.77)$ of the remaining heart rate trials were removed from each block. One participant was excluded from the respiratory rate analysis, as the stretch monitor sensor stopped working during the experiment. After excluding this participant, on average, 3.69\% $(\mathrm{SD}=0.014)$ of trials were removed from respiration trials. On average, $0.23 \%(\mathrm{SD}=0.32)$ of trials were removed from zygomaticus trials per block, and $0.57 \%(\mathrm{SD}=0.36)$ of trials were removed from corrugator trials per block. No skin conductance trials were removed.

\section{Results}




\section{PSYCHOPHYSIOLOGICAL RESPONSES TO AUDITORY CHANGE}

Overall performance for the boredom control task ranged from $84 \%$ to $100 \%$ across participants $(\mathrm{M}=97 \%, \mathrm{SD}=4 \%)$, indicating that participants were paying attention throughout the experiment. Erroneous trials were not removed, the aim of this supplementary task merely being to maintain a general state of vigilance.

To control for the potential effect of inter-participant variations in heart rate variability (HRV) on measures of heart rate, we first included RMSSD as a covariate in all repeatedmeasures ANCOVAs with heart rate as the dependent variable. The mean participant RMSSD was $50 \mathrm{~ms}(S D=43 \mathrm{~ms})$. However, RMSSD never had a significant effect on HR, and the obtained results never changed as a consequence of including RMSSD as a covariate, so we excluded HRV from all subsequent analyses.

Timbre Block. A significant Timbre $\times$ Epoch interaction on heart rate indicated that changes in timbre caused significant changes in heart rate over time, $F(12,456)=3.42, \varepsilon=.47$, $p=.004, \eta_{G}^{2}=.006$ (Figure 1A). There was a decrease in heart rate over time on trials without auditory change, indicated by the marginally significant decreasing linear trend in the Bassoon condition, $B=-.44, t(585)=-1.75, p=.080$. Changes to a Horn, Obochord or Trumpet timbre within the trial elicited a significant quadratic trend for each condition (Horn: $B=.66, t(585)=$ $2.51, p=.0122$; Obochord: $B=0.58, t(585)=2.00, p=.046$; Trumpet: $B=0.506, t(585)=2.27, p$ $=.023)$, indicating an initial decrease in heart rate in the first two seconds following the change, followed by an increase in the final two seconds of the recorded window. However, pair-wise polynomial trend comparisons indicated that only the linear trends demonstrated in the Trumpet and Obochord conditions differed significantly from the no-change condition $(p<.05)$. 


\section{PSYCHOPHYSIOLOGICAL RESPONSES TO AUDITORY CHANGE}

A

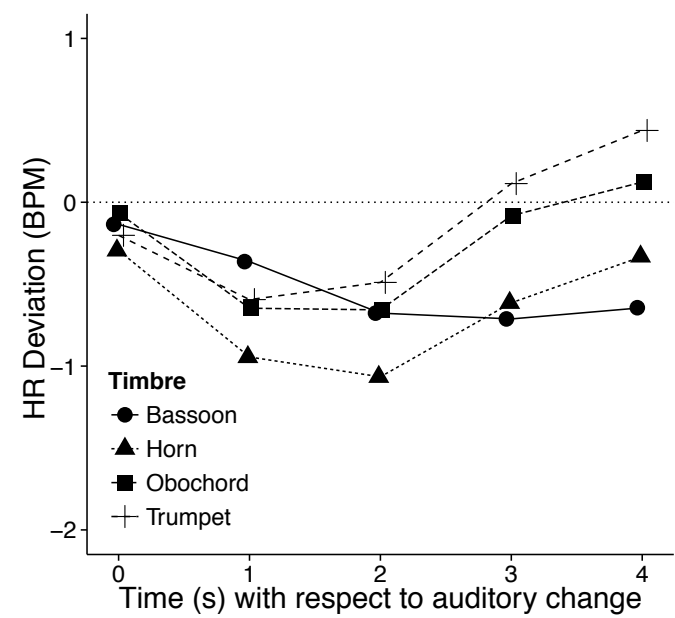

B

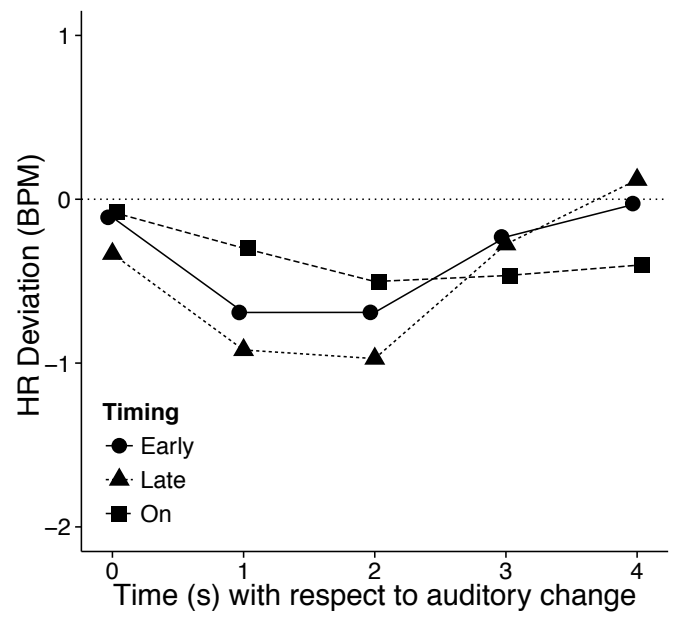

C

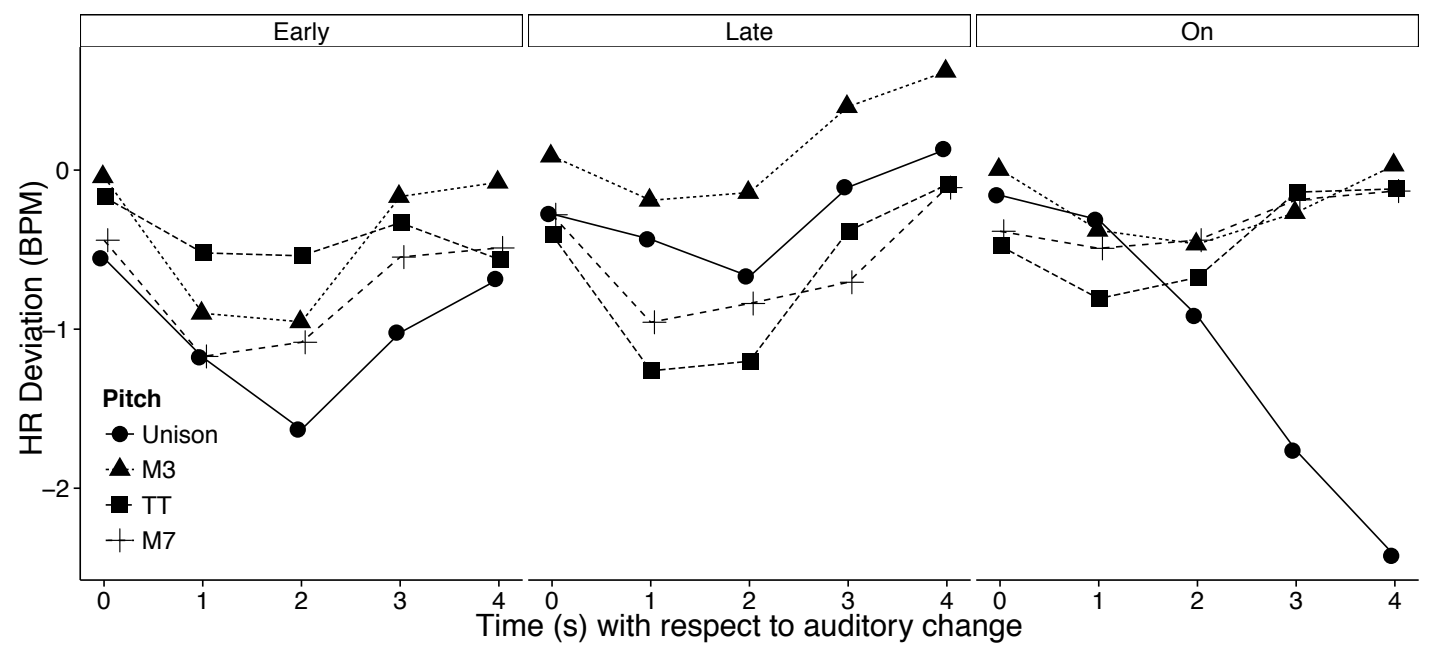

Figure 1. Experiment 1: Effect of changes in timbre (A) and rhythmic isochrony (B) on deviations from baseline heart rate (HR) (BPM=beats per minute). (C) Effect of pitch change on deviation from baseline heart rate in the Early, Late, and On-the-beat rhythmic isochrony conditions.

There was also a significant Isochrony $\mathrm{x}$ Epoch interaction on $\mathrm{HR}, F(8,304)=2.92$, $\varepsilon=.40, p=.034, \eta_{G}^{2}=.005$ (Figure 1B). HR decreased over time when there were no deviations 


\section{PSYCHOPHYSIOLOGICAL RESPONSES TO AUDITORY CHANGE}

in isochrony (On), indicated by a decreasing linear trend, $B=-0.32, t(780)=-2.21, p=.028$. Early and late deviations in isochrony both caused an initial decrease in HR followed by an increase, indicated by significant quadratic trends in both conditions (Early: $B=0.55, t(780)=$ 3.65, $p=.0003$; Late: $B=0.74, t(780)=5.12, p<.0001) . \quad$ Pair-wise polynomial trend comparisons indicated that the linear trend in the On condition was significantly different from the Late condition $(p<.05)$ and marginally different from the Early condition $(p=.093)$.

Finally, there was a significant effect of timbre on SCR magnitude, $F(3,117)=3.42$, $\varepsilon=.75, p=.032, \eta_{G}^{2}=.015($ Figure 2

A). Bonferroni-Holm adjusted, one-tailed comparisons indicated that only the Trumpet condition elicited a significantly greater SCR magnitude than the Bassoon condition $(p=.031)$. There was also a marginally significant main effect of rhythmic isochrony on SCR magnitude, $\mathrm{F}(2,78)=2.89, \varepsilon=0.78, p=.075, \eta_{G}^{2}=.008$ (Figure 2B). Adjusted one-tailed pairwise comparisons indicated that only the Late condition elicited a marginally greater SCR magnitude than the On condition $(p=.09)$.

A

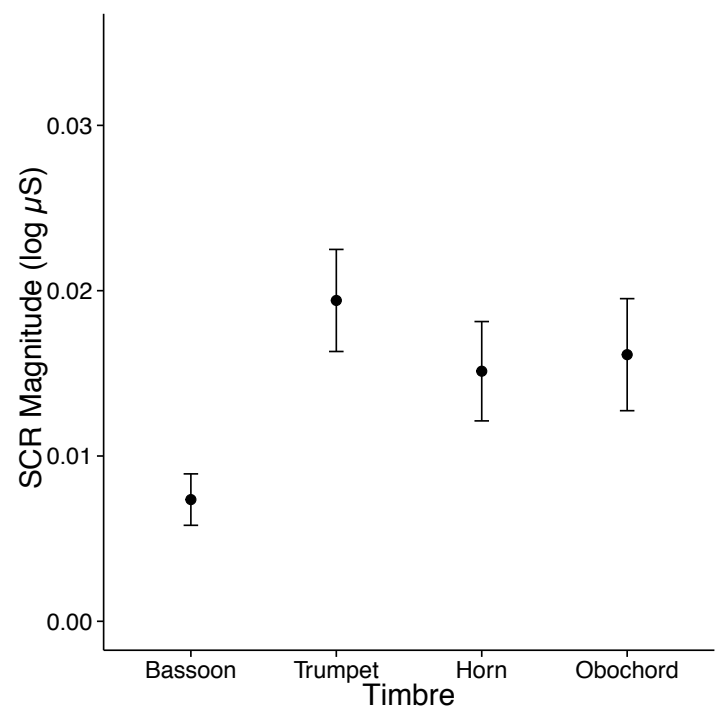

B

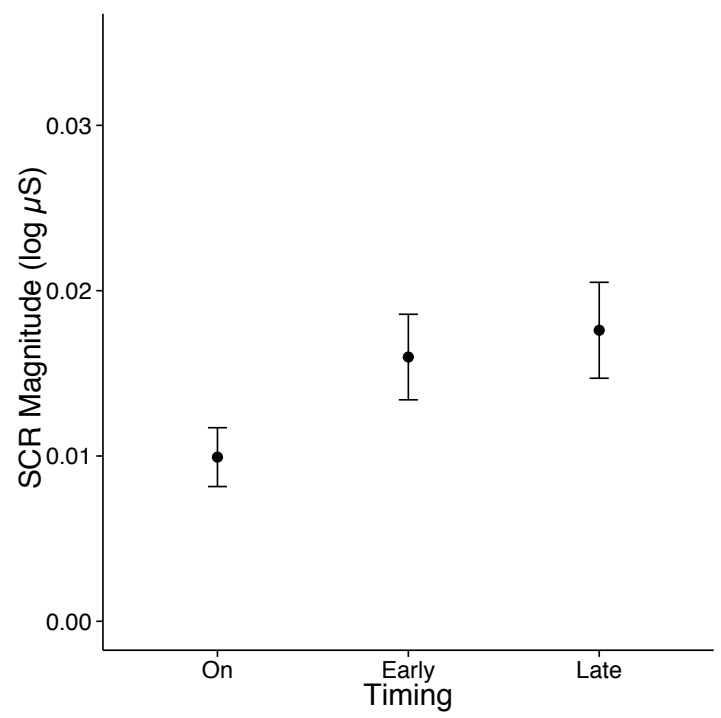




\section{PSYCHOPHYSIOLOGICAL RESPONSES TO AUDITORY CHANGE}

Figure 2. Experiment 1: (A) Effect of timbre change on SCR magnitude. (B) Effect of deviations from rhythmic isochrony on SCR magnitude in the timbre block. Error bars indicate 1 standard error about the mean.

There was no effect of isochrony or timbre on respiratory rate, proportion of inhalations during the moment of change, corrugator activity or zygomaticus activity.

Pitch Block. There was a significant three-way Pitch $\times$ Isochrony $\times$ Epoch interaction on heart rate, $F(24,912)=2.22, \varepsilon=0.35, p=.023, \eta_{G}^{2}=.008$, indicating that the effect of pitch change on heart rate over time was influenced by the rhythmic isochrony condition (Figure 1C). Although the linear trends for all pitch change conditions were significantly different from the Unison condition in the absence of rhythmic deviation $(p<.05)$, there were no significant polynomial trend differences between Unison and change conditions in both the Late and Early rhythmic deviation conditions. In isochronous (On) conditions, there was a decrease in HR when there was no pitch change, indicated by a decreasing linear trend, $B=-1.88, t(195)=-$ $5.55, p<.0001$, but there were no significant polynomial trends in all three pitch change conditions. Conversely, in the Early conditions, there was an initial decrease followed by an increase in HR in the Unison, M3 and M7 pitch conditions, indicated by quadratic trends for each condition (Early-Unison: $B=0.804, t(195)=3.80, p=.0002$; Early-M3: $B=0.74, t(195)=$ $3.36, p=.0010$. Early-M7: $B=0.56, t(195)=2.56, p=0.111)$. Similarly, for the Late condition, for both no-change and pitch-change conditions there was an initial decrease in HR followed by an increase in HR, indicated by significant quadratic trends for each (Late-Unison: $B=0.34$, $t(195)=2.09, p=.0382 ;$ Late-M3: $B=0.39, t(195)=1.77, p=.0787$; Late-TT: $B=0.83, t(195)$

$=3.90, p=.0001$; Late-M7: $B=0.58, t(195)=2.55, p=.0116)$. Finally, pair-wise trend 


\section{PSYCHOPHYSIOLOGICAL RESPONSES TO AUDITORY CHANGE}

comparisons indicated that in the isochronous condition, the linear trend for all three pitchchange conditions was significantly different $(\mathrm{p}<.05)$ from the Unison condition. However, for the Early and Late conditions there was no difference between linear trends for the changeconditions and the Unison condition.

Although there was no effect of pitch change on respiratory rate over time, deviations in isochrony elicited changes in the respiratory response (Figure 3), indicated by a marginally significant Epoch $\times$ Isochrony interaction, $F(8,304)=2.60, \varepsilon=0.25, p=.080, \eta_{G}^{2}=.002$. In the On condition, there was a decrease in respiratory rate, indicated by a decreasing linear trend, $B=$ $-0.251, t(195)=-5.45, p<.0001$. However, there were no significant polynomial trends for the Early and Late conditions, suggesting that respiration rate did not decrease over time when there were deviations in isochrony. Pairwise polynomial trend comparisons indicated that the linear trends for the On condition was marginally significantly different from those for the Early $(p<$ $.1)$ and Late $(p=.10)$ conditions.

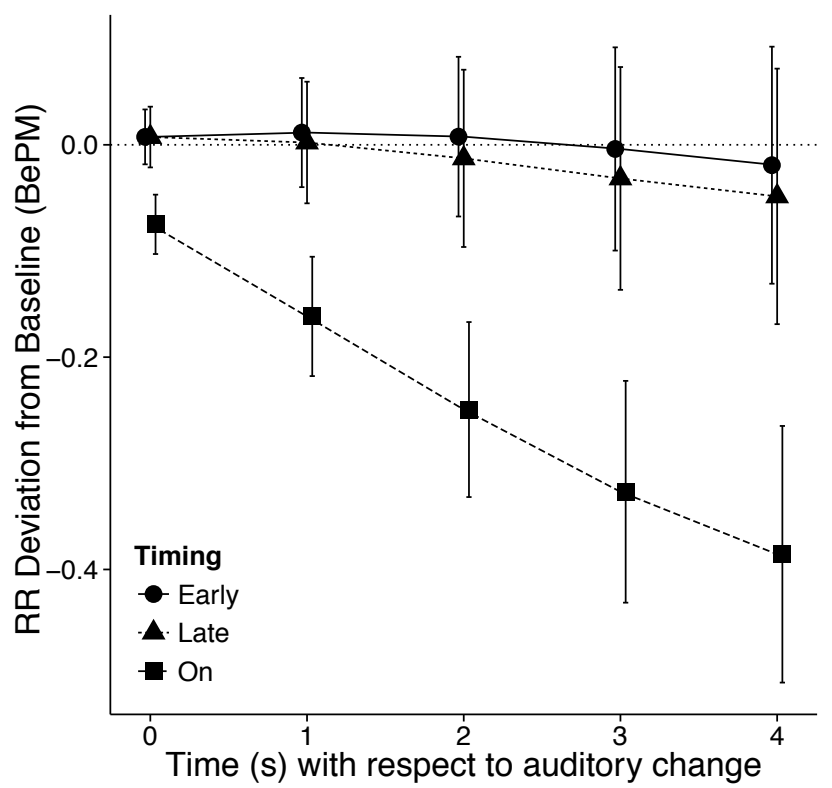




\section{PSYCHOPHYSIOLOGICAL RESPONSES TO AUDITORY CHANGE}

Figure 3. Experiment 1: Effect of deviations from rhythmic isochrony on respiratory rate $(\mathrm{RR})$ in the pitch block (BrPM=breaths per minute). Error bars indicate 1 standard error about the mean.

There was no effect of pitch or timing on SCR magnitude, zygomaticus activity, corrugator activity, or proportion of inhalations during the moment of change. 


\section{PSYCHOPHYSIOLOGICAL RESPONSES TO AUDITORY CHANGE}

\section{Discussion}

The results indicate that some autonomic physiological measures, but no somatic facial motor measures, were sensitive to simple changes in pitch, timbre, and rhythmic isochrony.

Heart rate was the most sensitive measure, responding to changes in all three parameters. When there was no change in timbre, pitch, or rhythmic isochrony, polynomial trend analysis indicated that on average, there was a decrease in heart rate over time, reflecting continual habituation to the repeating, standard stimulus. However, changes in timbre, pitch, and rhythmic isochrony all elicited a biphasic heart rate response, which consisted of an initial deceleration followed by a period of acceleration. Given that existing research has classified an initial heart rate deceleration followed by an acceleration back toward baseline as part of the orienting response (Graham \& Clifton, 1966; Öhman, Hamm, \& Hugdahl, 2000), results in the current experiment indicate that various changes in timbre, pitch and deviations from isochrony elicit the heart rate component of the orienting response.

SCR magnitude was also sensitive to auditory change. The SCR component of orienting was only observed in the timbre block, where changes in timbre and deviations from isochrony caused larger SCR magnitudes. However, post-hoc tests indicated that only the Trumpet timbre and Late deviation condition elicited a significantly larger SCR response compared to the notimbre change and isochronous conditions, respectively. Moreover, there was no effect of pitch, or an analogous effect of isochrony deviation in the pitch block on SCR.

Finally, although there were no changes in respiratory rate in response to changes in pitch or timbre, respiratory rate was sensitive to deviations in isochrony. In the pitch block, there was a decrease in respiratory rate over time in the isochronous condition, but no decrease was observed in either the Early or Late conditions. 


\section{PSYCHOPHYSIOLOGICAL RESPONSES TO AUDITORY CHANGE}

Although we predicted that the magnitude of the physiological response would be related to the degree of auditory change, the influence of individual timbre dimensions (spectral flux, spectral centroid, attack time) on autonomic response magnitude was not clear in the present experiment. The Trumpet timbre was the only timbre change that caused a significantly greater SCR magnitude from the no-change condition, and the Trumpet and Obochord timbres were the only changes that elicited significantly different heart rate trends. This finding might suggest that the orienting response is more sensitive to changes in spectral flux (the primary dimensional manipulation in the Trumpet condition) and attack time (the primary dimensional manipulation in the Obochord condition). However, given that we only included one timbre for each dimension of interest, we cannot draw any general conclusions about the relative influence of each dimension within the scope of this study. Nevertheless, to the best of our knowledge, the isolated effects of timbre change on autonomic physiology have not yet been examined, and we thus provide novel evidence that changes in timbre along various dimensions can affect some autonomic components of the orienting response.

Consistent with our predictions, deviations from rhythmic isochrony caused changes in autonomic components of the orienting response. Deviations from isochrony caused a pattern of deceleration followed by acceleration in heart rate relative to the isochronous condition in both the pitch and timbre blocks, changes in respiratory rate pattern in the pitch block, and increases in SCR magnitude in the timbre block. This finding provides evidence that disruptions of temporal expectations affect autonomic responding. However, similar to changes in timbre, given that there was only one degree of deviation in each temporal direction, we cannot draw general conclusions of the relative influence of early versus late deviations from isochrony. Interestingly, our finding that differences in the heart rate response as a function of pitch change 


\section{PSYCHOPHYSIOLOGICAL RESPONSES TO AUDITORY CHANGE}

were only present in the isochronous condition suggests that violations in isochrony may override the influence of pitch changes (in the Early and Late conditions).

Finally, although the degree of auditory change was clearly operationalized for changes in pitch, there did not appear to be a relationship between interval size and the magnitude of the heart rate response. This finding is consistent with previous work indicating that although heart rate is sensitive to pitch change, the degree of pitch change does not affect the magnitude of the heart rate response (Siddle \& Heron, 1976).

To extend our exploration to additional auditory parameters, we conducted a second experiment that assessed the effects of pitch, timbre, loudness, tempo, and duration changes on physiological response. We conducted Experiment 2 in the absence of a temporal isochrony manipulation to better isolate the effects of these parameters.

\section{Experiment 2}

\section{Method}

Participants. Forty participants (20 male; $M=21$ years, $S D=2$ years) took part in the experiment. None of the participants had taken part in Experiment 1. As in Experiment 1, they gave informed consent, completed a questionnaire on their musical experience, and had their hearing tested.

Stimuli. The stimulus sequence structure was identical to Experiment 1 with the following exceptions: (1) the number of events was dependent on the location of the target tone - the tone on which a change in auditory parameter occurs or not - to allow for 13 events to occur after the target tone, resulting in a response window of $8 \mathrm{~s}$ following the target. The auditory stimuli thus consisted of isochronous sequences of 24 to 28 events. The duration of the sequences ranged from 14 to $17 \mathrm{~s}$ to allow for a better analysis of respiration rate. (2) No 


\section{PSYCHOPHYSIOLOGICAL RESPONSES TO AUDITORY CHANGE}

sequences contained deviations from isochrony. (3) In addition to pitch and timbre variations, sequences contained variations in duration, tempo or sound level as summarized below.

Variations in loudness (sound level). All sequences were composed of 500-ms bassoonlike tones and contained an inter-onset interval (IOI) of $600 \mathrm{~ms}$. Six sequences contained no change in sound level (65 dB SPL), six sequences an increase to $70 \mathrm{~dB}$, six an increase to $75 \mathrm{~dB}$, and six an increase to $80 \mathrm{~dB}$.

Variations in duration. All sequences had inter-onset intervals of $600 \mathrm{~ms}$ between bassoon-like tones. Reference tones were $600 \mathrm{~ms}$ in duration, rather than $500 \mathrm{~ms}$, to allow for an adequate decrease in duration for change trials. Six sequences contained no change in duration from the reference tone, six a decrease to $450 \mathrm{~ms}$, six a decrease to $300 \mathrm{~ms}$, and six a decrease to $150 \mathrm{~ms}$ in duration.

Variations in tempo. To allow for an adequate amount of change within this parameter, reference tones had an IOI of $750 \mathrm{~ms}$ (80 BPM), and all bassoon-like tones were $300 \mathrm{~ms}$ in duration. Six sequences contained no change in IOI, six a decrease to an IOI of $600 \mathrm{~ms}$ (100 beats per minute), six a decrease to an IOI of $500 \mathrm{~ms}$ (120 beats per minute), and six a decrease to an IOI of $429 \mathrm{~ms}$ (140 beats per minute). A decrease in IOI creates an increase in tempo.

Procedure. The procedure was identical to Experiment 1 with the following exceptions. Each participant completed five experimental blocks of 24 trials. Each block was approximately 12 minutes in duration. Participants were presented with two practice trials before each block (no change, maximal change). An optional break was given after the first and third blocks. Participants were given the chance to notify the experimenter if they wished to pause.

Analyses. The analysis was identical to Experiment 1 with the following exception: for auditory change parameters where auditory change was increased in equal-sized intervals (i.e. 


\section{PSYCHOPHYSIOLOGICAL RESPONSES TO AUDITORY CHANGE}

intensity and tempo blocks), a polynomial trend analysis was conducted on the degree of change to assess whether SCR magnitude was related to the degree of auditory change. One participant's heart rate data were excluded due to consistent artifacts in the BVP signal. After excluding this participant, on average $4.3 \%$ of the remaining heart rate trials were removed from each block $(\mathrm{SD}=0.44 \%)$ due to signal artifacts. An average of $1.5 \%$ of the skin conductance trials were removed from each block $(\mathrm{SD}=0.4 \%)$. Two participants were also excluded from the zygomaticus activity analysis due to recurring artifacts in the signal, and one participant was excluded from the respiration rate analysis, again due to recurring artifacts in the signal.

\section{Results}

Aside from one participant who scored 37\%, overall performance for the boredomcontrol task ranged from $80 \%$ to $100 \%$ across participants $(M=94 \%, S D=5.3 \%)$, indicating that most participants were paying attention throughout the experiment. The participant with $37 \%$ performance accuracy was removed from the analysis, but otherwise erroneous trials were not removed.

To control for the potential effect of inter-participant variations in heart rate variability (HRV) on measures of heart rate, we again included RMSSD as a covariate in all repeatedmeasures ANCOVAs with heart rate as the dependent variable. The mean participant RMSSD was $55 \mathrm{~ms}(S D=55 \mathrm{~ms})$. As in Experiment 1, RMSSD never had a significant effect on HR, and the obtained results never changed, so we excluded HRV from all subsequent analyses.

Timbre Block. Changes in timbre caused changes in HR over time, indicated by a significant Timbre $\times$ Epoch interaction, $F(12,444)=4.06, \varepsilon=0.41, p=.002, \eta_{G}^{2}=.019$ (Figure 4A). When there was no auditory change, HR decreased over time, indicated by the negative linear trend in the Bassoon condition, $B=-1.19, t(190)=-3.02, p=.0029$. Conversely, the Horn 


\section{PSYCHOPHYSIOLOGICAL RESPONSES TO AUDITORY CHANGE}

timbre change caused an increase in HR over time, indicated by a significant increasing linear trend, $B=0.94, t(190)=2.43, p=.016$. The Obochord timbre change also caused an increase in HR after an initial deceleration, indicated by a significant quadratic trend, $B=0.69$, $t(190)=2.83, p=.005$. The Trumpet timbre change elicited a tri-phasic response; there was an initial decrease, followed by an increase, and then another decrease in HR, indicated by a marginally significant cubic trend, $B=-0.43, t(190)=-1.91, p=.057$. Finally, pair-wise linear trend comparisons indicated that the trend of the no-change condition for heart rate over time was significantly different from all change conditions $(p<.05)$. 


\section{PSYCHOPHYSIOLOGICAL RESPONSES TO AUDITORY CHANGE}

A

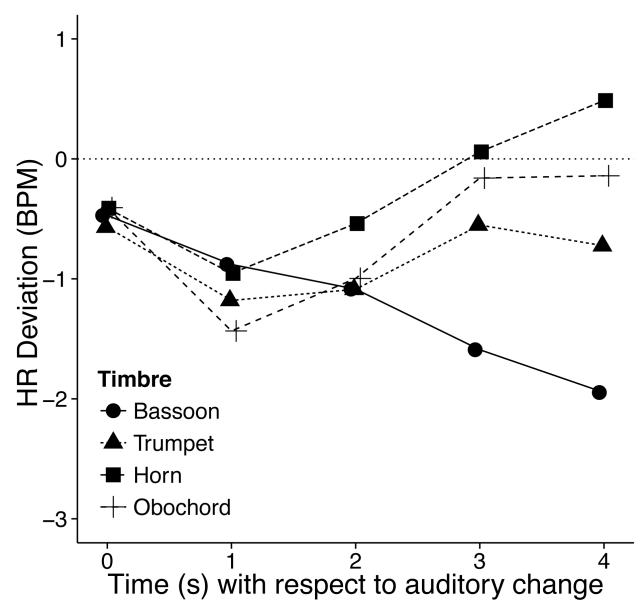

C

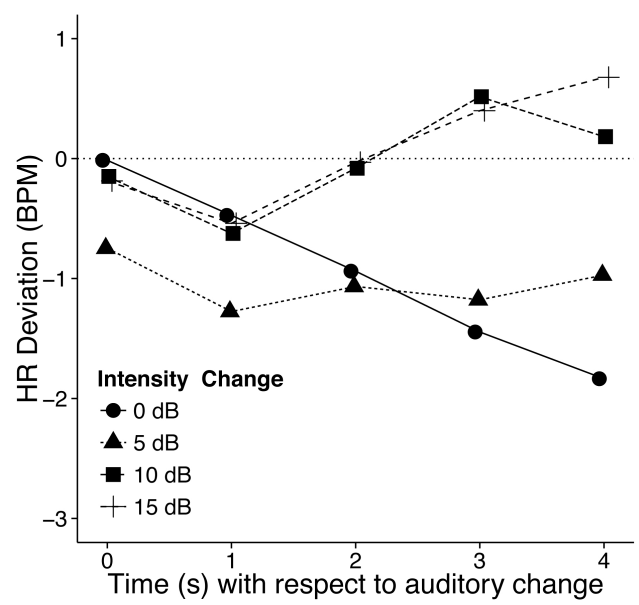

E

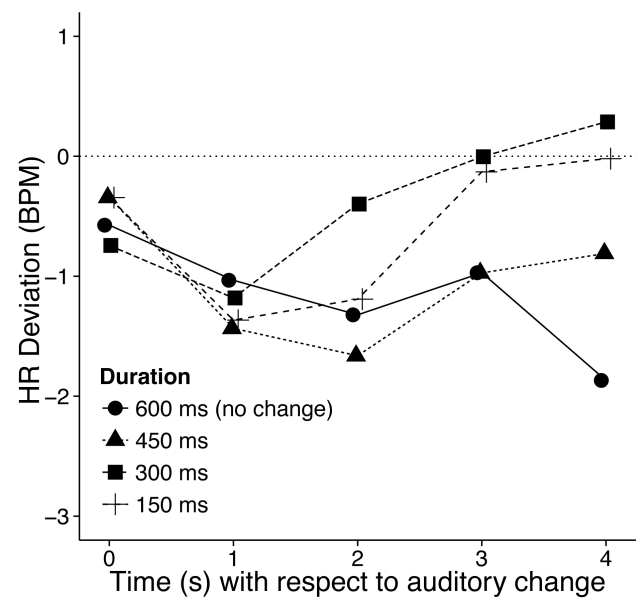

B

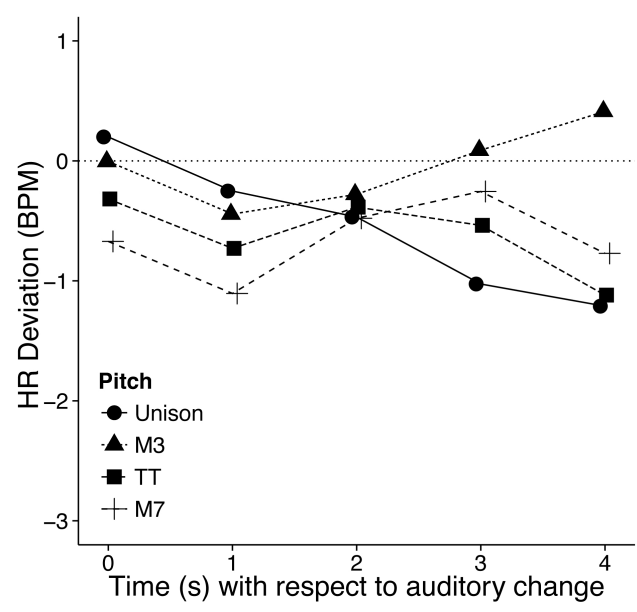

D

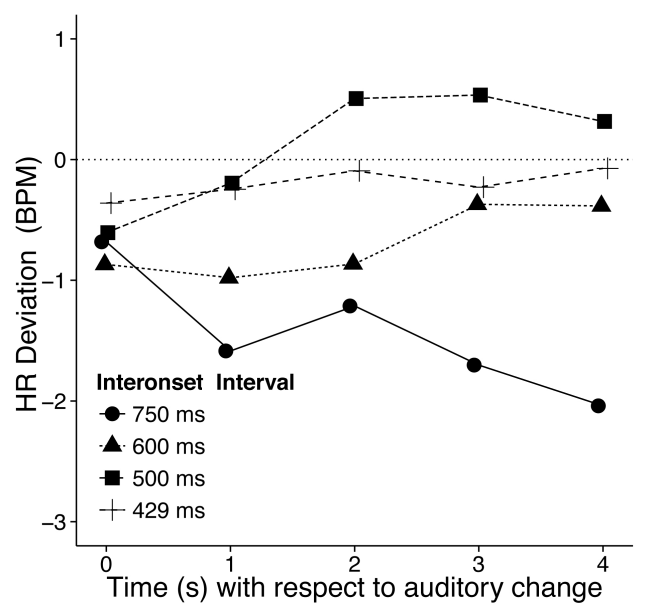

Figure 4. Experiment 2: Effect of changes in timbre (A), pitch (B), intensity (C), interonset interval (D), and tone duration (E) on deviation from baseline heart rate. 


\section{PSYCHOPHYSIOLOGICAL RESPONSES TO AUDITORY CHANGE}

Due to artifacts in the SCR signal, $0.9 \%$ of trials were excluded. Changes in timbre had a significant effect on SCR magnitude, $F(3,114)=3.17, p=.027, \eta_{G}^{2}=.043$, where changes in timbre elicited increases in SCR magnitude (Figure 5A). After Bonferroni-Holm corrections, one-tailed comparisons indicated that the Horn $(p=.098)$, Obochord $(p=.098)$ and Trumpet $(p=.080)$ conditions elicited a marginally significantly greater SCR magnitude than the nochange Bassoon condition. There was no effect of timbre on corrugator activity, zygomaticus activity, respiration rate, or proportion of inhalations during the moment of change. 


\section{PSYCHOPHYSIOLOGICAL RESPONSES TO AUDITORY CHANGE}

A

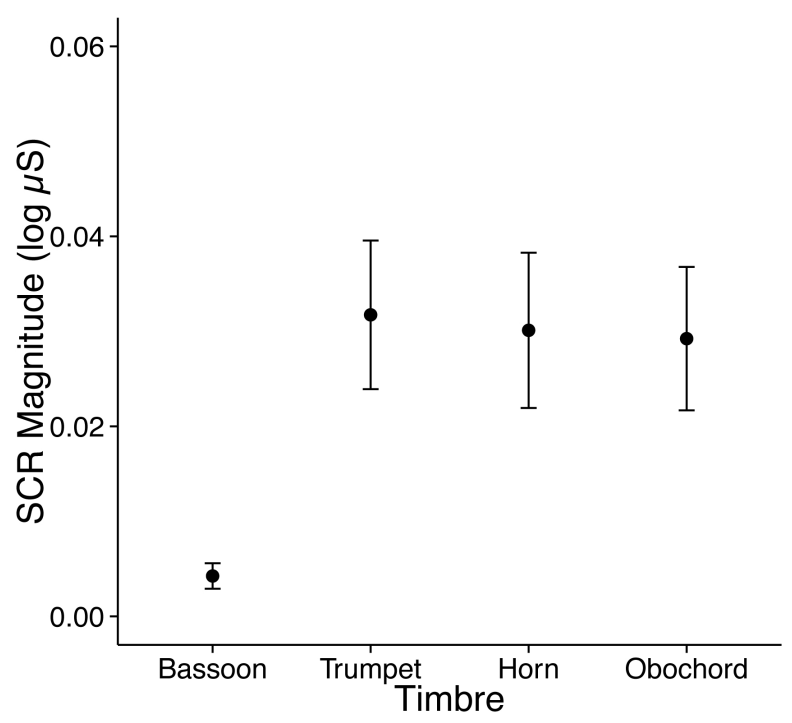

C

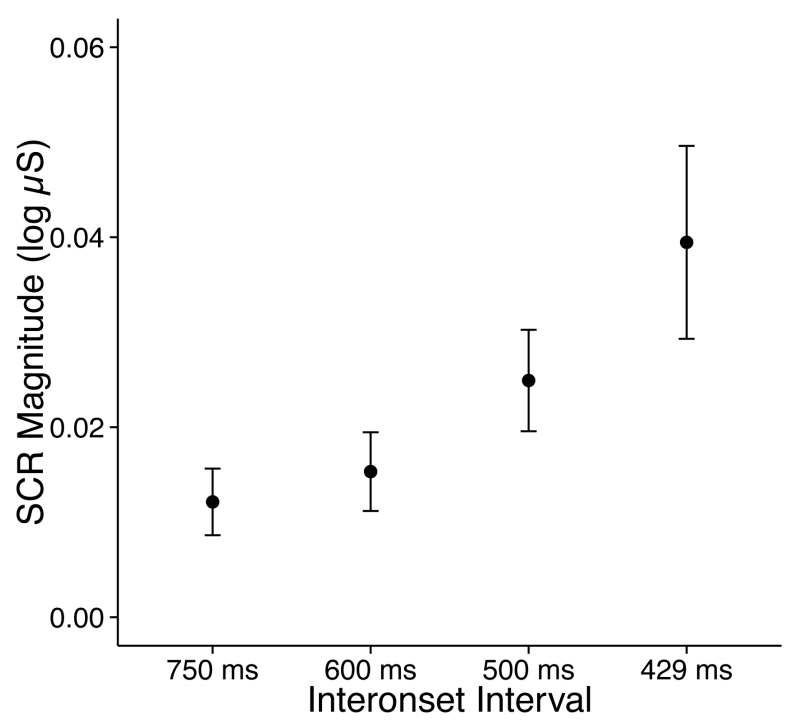

B

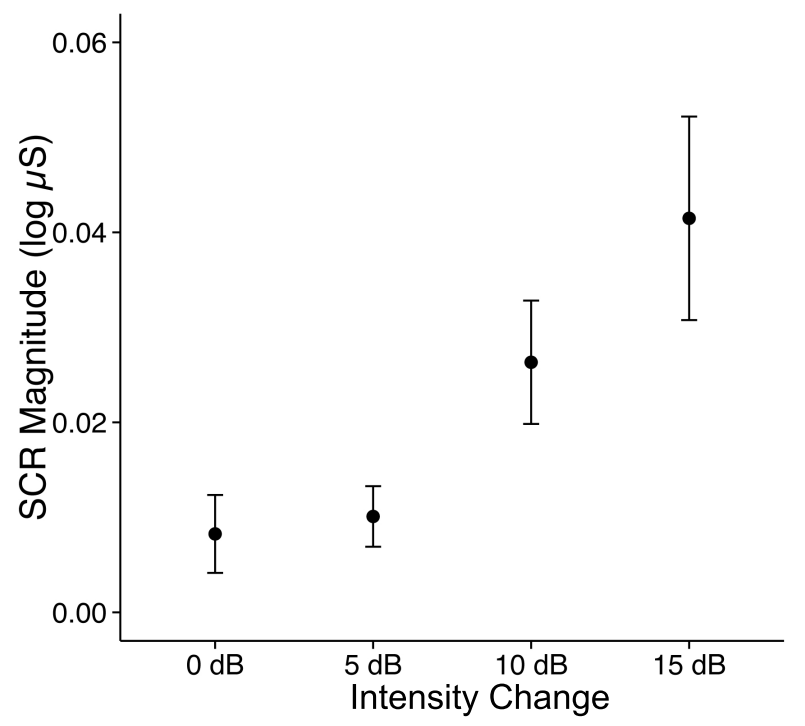

Figure 5. Experiment 2: Effect of changes in timbre (A), intensity (B), and tempo (C), on SCR magnitude. Error bars indicate 1 standard error about the mean.

Pitch Block. There was a marginally significant Pitch $\times$ Epoch interaction on heart rate, $F(12,444)=2.08, \varepsilon=.37, p=.078, \eta_{G}^{2}=.012$ (Figure 4B). When there was no auditory change, HR decreased over time, indicated by the negative linear trend in the Unison condition, 


\section{PSYCHOPHYSIOLOGICAL RESPONSES TO AUDITORY CHANGE}

$B=-1.17, t(190)=-3.12, p=.0021 . \quad$ A pitch increase of M3 caused an initial decrease, followed by increase in HR, indicated by a quadratic trend, $B=0.46, t(190)=2.05, p=.042$. Similarly, a pitch increase of M7 caused a tri-phasic HR response consisting of an initial decrease, followed by increase, then decrease in HR, which was indicated by a cubic trend, $B=-.60, t(190)=-2.79, p=.006$. Pair-wise linear trend comparisons indicated that only the major third and major seventh condition had significantly different trends compared to the nochange condition $(p<.05)$.

There was no effect of pitch change on SCR magnitude, corrugator activity, zygomaticus activity, respiration rate, or proportion of inhalations during the moment of change.

Intensity Block. There was a significant Intensity $\times$ Epoch interaction on heart rate, $F(12,444)=4.70, \varepsilon=0.45, p=.0003, \eta_{G}^{2}=.027$ (Figure 4C). When there was no auditory change, HR decreased over time, indicated by the negative linear trend in the $0 \mathrm{~dB}$ condition, $B=$ $-1.46, t(190)=-4.29, p<.0001$. Intensity increases of $10 \mathrm{~dB}$ caused a tri-phasic HR response consisting of an initial decrease, followed by increase, then decrease in HR, as indicated by a cubic trend, $B=-.61, t(190)=-3.06, p=.0026$. Intensity increases of $15 \mathrm{~dB}$ caused an increase in HR, indicated by an increasing linear trend, $B=.86, t(190)=2.41, p=.017$. Pair-wise linear trend comparisons indicated that the trend of the no-change condition for heart rate over time was significantly different from all change conditions $(p<.05)$.

There was a significant effect of intensity on SCR magnitude, $F(3,114)=3.30, \varepsilon=.66, p$ $=.043, \eta_{G}^{2}=.056$ (Figure 5B), where greater increases in intensity caused greater mean SCR magnitudes. A trend analysis conducted on intensity indicated a significant increasing quadratic trend; there is a non-linear relationship between intensity change and SCR magnitude $B=-0.026, t(156)=-2.92, p=.004$. Adjusted one-tailed pair-wise comparisons indicated that 


\section{PSYCHOPHYSIOLOGICAL RESPONSES TO AUDITORY CHANGE}

the $15 \mathrm{~dB}$ increase caused a significantly greater SCR magnitude than the no-change condition ( $p$ $=.036)$ and the $5-\mathrm{dB}$ condition $(p=.05)$.

There was no effect of intensity change on respiratory rate, corrugator activity, zygomaticus activity, or proportion of inhalations during the moment of change.

Tempo Block. There was a significant Tempo $\times$ Epoch interaction on heart rate, $F(12,444)=2.81, \varepsilon=0.47, p=.014, \eta_{G}^{2}=.015$ (Figure 4D). When there was no change in tempo, HR decreased over time, indicated by the negative linear trend in the $750 \mathrm{~ms}$ IOI condition, $B=-.897, t(190)=-2.45, p=.015$. An increase in tempo to a $500 \mathrm{~ms}$ IOI caused an increase in $\mathrm{HR}$, indicated by the increasing linear trend for the $500 \mathrm{~ms}$ condition, $B=.77$, $t(190)=2.02, p=.045$. There were no significant polynomial trends for the $429 \mathrm{~ms}$ and $600 \mathrm{~ms}$ conditions. Pair-wise linear trend comparisons indicated that the trend of the no-change condition for heart rate over time was significantly different from the $600 \mathrm{~ms}$ and $500 \mathrm{~ms}$ conditions $(p<.05)$ and only marginally significant for the $429 \mathrm{~ms}$ condition $(p=.085)$.

Due to artifacts in the skin conductance signal, $1.4 \%$ of trials were excluded from the analysis. There was a marginally significant effect of IOI change on SCR magnitude, $F(3,114)=$ $3.42, p=.051, \varepsilon=0.51, \eta_{G}^{2}=.050$ (Figure 5C). A trend analysis on tempo indicated a significant increasing linear trend, $B=.020, t(156)=2.67, p=.0084$, where greater increases in tempo (decreases in IOI) caused greater SCR magnitudes. After Bonferroni-Holm adjustments, onetailed pairwise comparisons indicated the largest IOI change (429 ms) elicited a significantly greater SCR than the $750 \mathrm{~ms}$, no-change condition $(p=.035)$, as well as a marginally significantly greater SCR compared to the $600 \mathrm{~ms}$ condition $(p=.063)$.

There was no effect of tempo on corrugator activity, zygomaticus activity, respiration rate, or proportion of inhalations during the moment of change 


\section{PSYCHOPHYSIOLOGICAL RESPONSES TO AUDITORY CHANGE}

Duration Block. There was a significant Duration $\times$ Epoch interaction on heart rate, $F(12,444)=3.98, \varepsilon=.527, p=.0006, \eta_{G}^{2}=.019$ (Figure 4E). When there was no change in tempo, HR decreased over time, as indicated by the marginally significant decreasing linear trend in the $600-\mathrm{ms}$ duration condition, $B=-.820, t(190)=-1.88, p=.062$. A change in duration to $450 \mathrm{~ms}$ elicited an initial decrease followed by increase in HR, indicated by a quadratic trend, $B=0.92, t(190)=4.83, p<.0001$. The $300-\mathrm{ms}$ duration change caused an increase in HR over time, indicated by an increasing linear trend, $B=1.01, t(190)=2.24, p=.027$. The largest change in duration $(150 \mathrm{~ms})$ caused an initial decrease and then increase in HR, indicated by a quadratic trend, $B=0.84, t(190)=3.96, p=.0001$. Pairwise comparisons indicated the linear trends for the $300 \mathrm{~ms}$ and $150 \mathrm{~ms}$ conditions were significantly different from the no-change condition $(p<.05)$, and that the quadratic trend for the $450 \mathrm{~ms}$ condition was different from the no-change condition $(p<.05)$.

There was also a main effect of duration on proportion of inhalations during the moment of auditory change, $F(3,114)=2.84, p=.041, \eta_{G}^{2}=.052$, where participants inhaled marginally more often in the $300 \mathrm{~ms}(p=.064)$ duration condition, relative to the $150 \mathrm{~ms}$ duration condition, after Bonferroni-Holm corrections for multiple comparisons. Note, however, that the no-change condition has the lowest HR deviation and the 300-ms condition has the highest deviation. There was no effect of duration on SCR magnitude, zygomaticus activity, corrugator activity, or respiration rate over time.

\section{Discussion}

The results of Experiment 2 suggest that in the absence of rhythmic irregularities, heart rate and skin conductance components of the orienting response are sensitive to simple changes in timbre, pitch, intensity, tempo, and duration. Facial motor activity and respiratory rate were 


\section{PSYCHOPHYSIOLOGICAL RESPONSES TO AUDITORY CHANGE}

not affected by auditory change. Heart rate was the most sensitive measure, responding to change for all parameters except pitch (which had a marginally significant effect). There was generally a decrease in heart rate over time when there was no auditory change and some combination of heart rate deceleration and/or acceleration in response to auditory change. SCR magnitude was sensitive to changes for all parameters except pitch and duration. For parameters where a polynomial trend analysis was conducted on the degree of change (i.e. intensity and tempo), results indicated that the magnitude of the SCR was proportional to the degree of auditory change.

Changes in timbre affected both the heart rate response and SCR magnitude. All timbre changes caused heart rate responses that differed from the continuous deceleration of the nochange condition. Trend analyses indicated an overall heart rate acceleration for the Horn condition, and an initial deceleration followed by an acceleratory component for the Trumpet and Obochord conditions. All changes in timbre caused larger SCR magnitudes compared to the nochange condition, although there were no differences between conditions. Given that we only included one timbre for each dimension of interest, and that there were no differences between the three timbre-change conditions, as in Experiment 1, we again could not draw firm conclusions regarding the relative influence of individual timbre dimensions. Acoustic changes in perceptually relevant timbre dimensions, such as spectral centroid (Toiviainen et al., 1998) and rise time (Lyytinen et al., 1992) have been shown to cause the MMN, and research suggests that different timbre dimensions may be processed separately in auditory sensory memory (Caclin et al., 2006). Our findings add to the body of work on the neural correlates of timbre change by indicating that changes in timbre also affect autonomic responding. Future work could lend insight into the influence of timbre change on autonomic responding by exploring a wider 


\section{PSYCHOPHYSIOLOGICAL RESPONSES TO AUDITORY CHANGE}

variety of timbres, as well as by considering the role of timbre saliency, a quality referring to how well a timbre captures one's attention (Chon \& McAdams, 2012).

Similar to the results of Experiment 1, increases in pitch elicited heart rate but not SCR components of the orienting response. Interestingly, only the 4-semitone (M3) and 11-semitone (M7) pitch-increase conditions elicited heart rate responses that differed significantly from the continuous deceleration of the no-change condition: both involved an initial deceleration, followed by an acceleration in heart rate (and an additional deceleration in the M7 condition). Given that the M3 is a smaller interval than the TT (6 semitones), this finding is contrary to our prediction that the physiological response would reflect the size of the pitch change. Moreover, the null effects of pitch change on SCR magnitude in this experiment are inconsistent with previous findings, which have shown that larger SCRs are elicited by larger changes in pitch (Siddle \& Heron, 1976). Our null effects on SCR may thus be the result of the limited pitch range we employed in this study. Whereas Siddle \& Heron (1976) investigated decreases in pitch from $1000 \mathrm{~Hz}$ to either $670 \mathrm{~Hz}$ (smaller pitch change, 8.3 semitones) or $380 \mathrm{~Hz}$ (larger change, 22.7 semitones), our largest pitch change was an increase from $311 \mathrm{~Hz}$ to $659 \mathrm{~Hz}$ (11 semitones), as this range corresponds more closely to the successive pitch intervals observed in music.

Consistent with previous research on orienting, we found that increases in stimulus intensity affected the heart rate response and SCR magnitude. Whereas there was a decrease in heart rate in the no-change condition, the $10 \mathrm{~dB}$ increase caused an initial deceleration, followed by heart rate acceleration, and the $15 \mathrm{~dB}$ increase elicited an overall acceleratory response. These results might suggest that although smaller increases in intensity elicit an orienting response (indicated by an early decelerating component), larger intensity changes elicit a defensive response (primarily an acceleration in heart rate). Increases in intensity also caused increases in 


\section{PSYCHOPHYSIOLOGICAL RESPONSES TO AUDITORY CHANGE}

SCR magnitude. Trend analysis indicated a quadratic trend of intensity change on SCR magnitude, suggesting that the size of the SCR magnitude is proportional to the amount of intensity decibel increase. These findings corroborate research indicating that differences in stimulus intensity affect cardiovascular and electrodermal activity (Turpin, Schaefer, \& Boucsein, 1999).

Increases in tempo caused changes in the heart rate response and skin conductance response. Heart rate decreased over time when there was no change in tempo, and increased over time in response to the $500 \mathrm{~ms}$ IOI change. Interestingly, for the 429-ms and 600-ms IOI conditions, there were no significant polynomial trends, suggesting that there was neither acceleration nor deceleration in heart rate for these conditions, although these responses still differed significantly from the deceleration of the no-change condition. These results suggest that there is no clear relationship between the nature of the heart rate response and the degree of tempo change. For the skin conductance component of orienting, however, the effect of tempo change on SCR magnitude was more apparent. Increases in tempo were linearly proportional to SCR magnitude, where larger tempo changes caused larger SCR magnitudes. Overall, our findings can be related to previous research on music listening, which shows that increases in musical tempo cause increases in heart rate and skin conductance level (Gomez \& Danuser, 2007). However, little research has been conducted on the link between tempo and physiology outside the context of music listening, and as such, our findings provide novel evidence that an increase in tempo can elicit autonomic components of the orienting response.

Lastly, decreases in tone duration affected the heart rate response but did not affect SCR magnitude. Although HR decreased over time when there was no change in duration, trend analyses indicated that the 450 -ms and 150 -ms duration changes caused an initial deceleration 


\section{PSYCHOPHYSIOLOGICAL RESPONSES TO AUDITORY CHANGE}

followed by acceleration in heart rate. Although it was not the largest decrease in duration, the 300-ms duration condition elicited an overall increase in heart rate over time. These results might suggest that there is no clear relationship between the heart rate response and the degree of duration change, or alternatively, that it may be partially explained by the respiratory data. Participants tended to inhale more often on the no-change and 300-ms conditions, which may have exaggerated the heart rate response in the 300 -ms condition, although we did not observe an exaggerated heart rate response in the no-change condition. Our findings on duration can be compared to previous research on orienting assessing longer duration decrements (from $15 \mathrm{~s}$ stimulus to $5 \mathrm{~s}$ ), in which there is also no effect of auditory duration change on the SCR (Siddle, Kyriacou, \& Heron, 1978). Moreover, our findings are consistent with existing research indicating that human listeners are sensitive to changes in shorter auditory duration ranges, where decreases in auditory duration elicit the MMN in the ERP signal (Näätänen, Paavilainen, \& Reinikainen, 1989).

As a final note, effect sizes were somewhat larger overall for SCR magnitude relative to heart rate and respiration rate in Experiment 2. Rather than necessarily indicating a greater effect of auditory change on skin conductance than the other autonomic measures, however, this difference can likely be attributed to the fact that there were more independent factors for epochbased analyses (as they included the Epoch factor). This point is unsurprising given that the effect size of a particular factor generally decreases as the number of overall factors causing the effect increases (Ahadi \& Diener, 1989; Levine \& Hullett, 2002). A similar interpretation applies to the discrepancy between effect sizes of auditory change on heart rate in Experiments 1 and 2. Whereas the effect size of timbre and pitch change on heart rate in Experiment 1 seems 


\section{PSYCHOPHYSIOLOGICAL RESPONSES TO AUDITORY CHANGE}

miniscule in comparison to the effect size of these parameters in Experiment 2, this result can likely be attributed to the addition of isochrony as a factor in the first experiment. 


\section{PSYCHOPHYSIOLOGICAL RESPONSES TO AUDITORY CHANGE}

\section{General Discussion}

The results of the current study extend our understanding of the relationship between auditory processing and autonomic response patterns. Previous research using the MMN as an index of auditory change detection has shown that auditory processing is sensitive to changes in pitch (Jacobsen, Schröger, Horenkamp, \& Winkler, 2003), duration (Näätänen et al., 1989), timbre (Caclin et al., 2006), and intensity (Jacobsen, Horenkamp, \& Schröger, 2003). This study provides evidence that these parameters, along with other musically relevant parameters such as tempo and timbre, can also elicit measurable changes in autonomic response patterns. Heart rate was sensitive to changes in all parameters (isochrony, pitch, timbre, loudness, tempo, duration), SCR magnitude was sensitive to timbre, loudness, and tempo, and respiratory rate was sensitive to deviations from isochrony. We did not find any effects of auditory change on zygomaticus or corrugator EMG activity.

Interestingly, our findings in some ways align with research investigating the structural properties of music that underlie what are thought to be emotion-related physiological changes. We demonstrate that physiological responses elicited by musical stimuli can also be caused by similar acoustic changes in controlled, simple sequences. For example, our finding that deviations from isochronous regularity caused changes in heart rate can be related to Sloboda's (1991) research on self-reported heart racing, which was associated with musical syncopation (unexpected or offbeat rhythms in music) and unexpected, early occurrences of musical events. We also found that tempo increases caused increases in SCR magnitude, and in some conditions, increases in heart rate over time. Comparable relationships between tempo and physiology have been found during music listening: happy musical excerpts with fast tempi elicit greater SCR magnitudes than sad excerpts with slow tempi (Khalfa, Peretz, Blondin, \& Robert, 2002), and 


\section{PSYCHOPHYSIOLOGICAL RESPONSES TO AUDITORY CHANGE}

faster musical tempi have been linearly associated with higher skin conductance level and heart rate (Gomez \& Danuser, 2007).

In both experiments, we observed increases in SCR magnitude and changes in heart rate in response to timbre changes. Similarly, increases in percussiveness in music, a property related to the attack-decay components of timbre, have been linked to increases in skin conductance level (van der Zwaag, Westerink, \& van den Broek, 2011). Finally, a comprehensive investigation of low-level acoustic parameters relating music, emotion, and physiology found a linear relation between loudness, tempo, pitch level, and timbre ("sharpness") and subjective ratings of emotional arousal, which were in turn correlated with increases in heart rate (Coutinho \& Cangelosi, 2011). Again, our research suggests that changes in loudness, tempo, pitch and timbre also cause acceleration in heart rate in the context of simple, controlled auditory sequences.

Taken together, these data present important methodological considerations for researchers interested in using physiological measures to index emotion. It is clear that many of the physiological changes displayed during music listening that reflect autonomic nervous system activity — which are often reported as evidence of emotion induction — can also be elicited by simple acoustic sequences that are unlikely to elicit emotional responses. These results may therefore suggest that physiological responses elicited during music listening are just as likely to index orienting and attention as they are to reflect genuine emotion episodes (e.g., happiness, anger or fear). Indeed, the absence of any significant variations in facial muscle activity witnessed in these studies provides evidence in support of this view, since the zygomaticus and corrugator muscles often serve as robust indicators of positive and negative affect, respectively. 


\section{PSYCHOPHYSIOLOGICAL RESPONSES TO AUDITORY CHANGE}

However, these findings do not rule out the possibility that simple auditory changes could induce emotional responses. The stimuli in this study may have elicited defensive responses, which are typically associated with negative affect, and some researchers have even suggested a link between orienting and emotion (for a review of this topic, see Bradley, 2009). We thus cannot definitively conclude that our current stimuli were non-emotional: only that they were much simpler auditory sequences that elicited similar physiological responses to those found in other music listening studies. That being said, a classic marker of negative affect, corrugator response, was absent from any of our stimuli.

It is worthwhile to mention some limitations in the present study, which stem primarily from the scope of auditory change examined. First, on most acoustic parameters, only unidirectional change was examined (e.g., only increases in loudness or decreases in duration). We therefore cannot draw any conclusions about the effect of directionality of change from the physiological responses measured here, although there may be important asymmetries in the response profile for acoustic changes in parameters like loudness, where sudden increases are more likely to orient listeners to the auditory stimulus than sudden decreases. Second, we chose to explore acoustic changes on magnitudes resembling those that occur in a musical context, thereby constraining our investigation to relatively small degrees of change. As a result, this constraint may have obscured potential relationships between auditory change magnitude and physiological response magnitude for parameters like pitch, where fewer effects emerged in the experiments reported here. Finally, it was difficult to make meaningful comparisons between different parameters, as the perceived magnitude of the range of change was not equalized between blocks. It is thus possible that there were fewer effects observed in the pitch block not 


\section{PSYCHOPHYSIOLOGICAL RESPONSES TO AUDITORY CHANGE}

because changes in pitch are less salient, but because the largest pitch change was perceived as smaller in magnitude than the largest loudness change.

Nonetheless, our findings highlight the importance of using converging methods to accurately capture emotional responses during music listening: to collect physiological measurements in combination with subjective ratings of emotion induction. This approach would be consistent with dominant accounts of emotion theory that describe affective changes as a multi-component construct involving both changes in subjective feeling and physiology (Bradley \& Lang, 2000b; Juslin \& Västfjäll, 2008; Mauss, Levenson, McCarter, Wilhelm, \& Gross, 2005; Scherer, 2005), and would strengthen the validity of claims made about the music-emotion link.

\section{References}

Ahadi, S., \& Diener, E. (1989). Multiple determinants and effect size. Journal of Personality and Social Psychology, 56(3), 398-406. doi:10.1037/0022-3514.56.3.398

Bakeman, R. (2005). Recommended effect size statistics for repeated measures designs. Behavior Research Methods, 37(3), 379-384. doi:10.3758/bf03192707

Barry, R., \& Maltzman, I. (1985). Heart rate deceleration is not an orienting reflex: Heart rate acceleration is not a defensive reflex. The Pavlovian Journal of Biological Science, 20(1), $15-28$.

Barry, R. J., \& Sokolov, E. N. (1993). Habituation of phasic and tonic components of the orienting reflex. International Journal of Psychophysiology, 15(1), 39-42. doi:10.1016/0167-8760(93)90093-5 


\section{PSYCHOPHYSIOLOGICAL RESPONSES TO AUDITORY CHANGE}

Berntson, G. G., Quigley, K. S., \& Lozano, D. (2007). Cardiovascular psychophysiology. In J. T. Cacioppo, L. G. Tassinary \& G. G. Berntson (Eds.), The handbook of psychophysiology (3rd ed., pp. 182-210). New York, NY: Cambridge University Press.

Boucsein, W. (2012). Electrodermal activity (2nd ed.). New York, NY: Springer. doi: 10.1007/978-1-4614-1126-0

Bradley, M. (2009). Natural selective attention: Orienting and emotion. Psychophysiology, 46(1), 1-11. doi:10.1111/j.1469-8986.2008.00702.x

Bradley, M., \& Lang, P. (2000a). Affective reactions to acoustic stimuli. Psychophysiology, 37(2), 204-215, doi:10.1111/1469-8986.3720204

Bradley, M., \& Lang, O. (2000b). Measuring emotion: Behavior, feeling, and physiology. In R. D. Lane, L. Nadel, \& G. Ahern (Eds.). Cognitive neuroscience of emotion (pp. 242-276). New York, NY: Oxford University Press.

Caclin, A., Brattico, E., Tervaniemi, M., Näätänen, R., Morlet, D., Giard, M.H., \& McAdams, S. (2006). Separate neural processing of timbre dimensions in auditory sensory memory. Journal of Cognitive Neuroscience, 18(12), 1959-1972. doi:10.1162/jocn.2006.18.12.1959

Chon, S. H., \& McAdams, S. (2012). Investigation of timbre saliency, the attention-capturing quality of timbre. The Journal of the Acoustical Society of America, 131(4), 3433-3433. doi: $10.1121 / 1.4708879$

Cook, E., \& Turpin, G. (1997). Differentiating orienting, startle, and defense responses: The role of affect and its implications for psychopathology. In P. J. Lang, R.F. Simons, \& M. Balaban (Eds.). Attention and orienting: Sensory and motivational processes (pp. 137164). Hove, UK: Psychology Press. 


\section{PSYCHOPHYSIOLOGICAL RESPONSES TO AUDITORY CHANGE}

Coutinho, E., \& Cangelosi, A. (2011). Musical emotions: Predicting second-by-second subjective feelings of emotion from low-level psychoacoustic features and physiological measurements. Emotion, 11(4), 921-937. doi:10.1037/a0024700

Gati, I., \& Ben-Shakhar, G. (1990). Novelty and significance in orientation and habituation: A feature matching approach. Journal of Experimental Psychology: General, 119(3), 251263. doi:10.1016/0167-8760(91)90065-6

Gomez, P., \& Danuser, B. (2007). Relationships between musical structure and psychophysiological measures of emotion. Emotion, 7(2), 377-387. doi:10.1037/15283542.7 .2 .377

Graham, F. K., \& Clifton, R. K. (1966). Heart-rate change as a component of the orienting response. Psychological Bulletin, 65(5), 305-320. doi:10.1037/h0023258

ISO 389-8. (2004). Acoustics: Reference zero for the calibration of audiometric equipment. Part 8: Reference equivalent threshold sound pressure levels for pure tones and circumaural earphones. (Tech. Rep.). Geneva, Switzerland: International Organization for Standardization.

Jackson, J. C. (1974). Amplitude and habituation of the orienting reflex as a function of stimulus intensity. Psychophysiology, 11(6), 647-659.

Jacobsen, T., Schröger, E., Horenkamp, T., \& Winkler, I. (2003). Mismatch negativity to pitch change: Varied stimulus proportions in controlling effects of neural refractoriness on human auditory event-related brain potentials. Neuroscience Letters, 344(2), 79-82.

Jacobsen, T., Horenkamp, T., \& Schröger, E. (2003). Preattentive memory-based comparison of sound intensity. Audiology and Neuro-Otology, 8(6), 338-346. doi:10.1159/000073518 


\section{PSYCHOPHYSIOLOGICAL RESPONSES TO AUDITORY CHANGE}

Juslin, P. N., \& Västfjäll, D. (2008). Emotional responses to music: The need to consider underlying mechanisms. Behavioral and Brain Sciences, 31(5), 559-575.

Khalfa, S., Peretz, I., Blondin, J.-P., \& Robert, M. (2002). Event-related skin conductance responses to musical emotions in humans. Neuroscience Letters, 328(2), 145-149. doi:10.1016/s0304-3940(02)00462-7

Krimphoff, J., McAdams, S., \& Winsberg, S. (1994). Caractérisation du timbre des sons complexes. II. Analyses acoustiques et quantification psychophysique [Characterization of the timbre of complex sounds. II. Acoustic analyses and psychophysical quantification]. Le Journal de Physique, 4(C5), 625-628.

Large, E. W., \& Jones, M. R. (1999). The dynamics of attending: How people track time-varying events. Psychological Review, 106(1), 119-159. doi:10.1037/0033-295x.106.1.119

Levine, T. R., \& Hullett, C. R. (2002). Eta squared, partial eta squared, and misreporting of effect size in communication research. Human Communication Research, 28(4), 612625. doi:10.1111/j.1468-2958.2002.tb00828.x

Lyytinen, H., Blomberg, A.-P., \& Näätänen, R. (1992). Event-related potentials and autonomic responses to a change in unattended auditory stimuli. Psychophysiology, 29(5), 523-534. doi:10.1111/j.1469-8986.1992.tb02025.x

Madison, G., \& Merker, B. (2004). Human sensorimotor tracking of continuous subliminal deviations from isochrony. Neuroscience Letters, 370(1), 69-73. doi:10.1016/j.neulet.2004.07.094

Martin, F. N., \& Champlin, C. A. (2000). Reconsidering the limits of normal hearing. Journal of the American Academy of Audiology, 11(2), 64-66. 


\section{PSYCHOPHYSIOLOGICAL RESPONSES TO AUDITORY CHANGE}

Mauss, I. B., Levenson, R. W., McCarter, L., Wilhelm, F. H., \& Gross, J. J. (2005). The tie that binds? Coherence among emotion experience, behavior, and physiology. Emotion, 5(2), 175-190. doi:10.1037/1528-3542.5.2.175

McAdams, S., Winsberg, S., Donnadieu, S., Soete, G., \& Krimphoff, J. (1995). Perceptual scaling of synthesized musical timbres: Common dimensions, specificities, and latent subject classes. Psychological Research, 58(3), 177-192. doi:10.1007/bf00419633

McAuley, D. J., \& Kidd, G. R. (1998). Effect of deviations from temporal expectations on tempo discrimination of isochronous tone sequences. Journal of Experimental Psychology: Human Perception and Performance, 24(6), 1786-1800. doi:10.1037/00961523.24.6.1786

Müller, M. (2007). Information retrieval for music and motion. New York, NY: Springer-Verlag. Näätänen, R., Paavilainen, P., \& Reinikainen, K. (1989). Do event-related potentials to infrequent decrements in duration of auditory stimuli demonstrate a memory trace in man? Neuroscience Letters, 107(1-3), 347-352. doi:10.1016/0304-3940(89)90844-6

Näätänen, R., \& Winkler, I. (1999). The concept of auditory stimulus representation in cognitive neuroscience. Psychological Bulletin, 125(6), 826-859. doi:10.1037/00332909.125.6.826

Öhman, A., Hamm, A., \& Hugdahl, K. (2000). Cognition and the autonomic nervous system: Orienting, anticipation, and conditioning. In J. T. Cacioppo, L. G. Tassinary, \& G. G. Berntson (Eds.). Handbook of psychophysiology (2nd ed., pp. 533-575). Cambridge, UK: Cambridge University Press.

Olejnik, S., \& Algina, J. (2003). Generalized eta and omega squared statistics: measures of effect size for some common research designs. Psychological Methods, 8(4), 434. 


\section{PSYCHOPHYSIOLOGICAL RESPONSES TO AUDITORY CHANGE}

Ooishi, Y., \& Kashino, M. (2012). Habituation of rapid sympathetic response to aversive timbre eliminated by change in basal sympathovagal balance. Psychophysiology. 49(8), 10591071. doi:10.1111/j.1469-8986.2012.01393.x

Open Sound Control (n.d.). opensoundcontrol.org (last accessed April 13, 2015).

Scherer, K. R. (2005). What are emotions? And how can they be measured? Social Science Information, 44(4), 695-729.

Smith, B. K. (1995). PsiExp: An environment for psychoacoustic experimentation using the IRCAM Musical Workstation. Paper presented at the Society for Music Perception and Cognition Conference, University of California, Berkeley, California.

Siddle, D. A. (1991). Orienting, habituation, and resource allocation: An associative analysis. Psychophysiology, 28(3), 245-259.

Siddle, D. A. , \& Heron, P. A. (1976). Effects of length of training and amount of tone frequency change on amplitude of autonomic components of the orienting response. Psychophysiology, 13(4), 281-287.

Siddle, D. A. T., Kyriacou, C., \& Heron, P. A. (1978). Effects of change in stimulus duration on amplitude of the electrodermal orienting response. Physiological Psychology, 6(1), 121125. doi:10.3758/bf03326705

Sloboda, J. A. (1991). Music structure and emotional response: Some empirical findings. Psychology of Music, 19(2), 110-120. doi:10.1177/0305735691192002

Sokolov, E. N. (1963). Perception and the conditioned reflex. Oxford, UK: Pergamon.

Stekelenburg, J. J., \& van Boxtel, A. (2002). Pericranial muscular, respiratory, and heart rate components of the orienting response. Psychophysiology, 39(6), 707-722.

doi:10.1111/1469-8986.3960707 


\section{PSYCHOPHYSIOLOGICAL RESPONSES TO AUDITORY CHANGE}

Tiitinen, H., May, P., Reinikainen, K., \& Näätänen, R. (1994). Attentive novelty detection in humans is governed by pre-attentive sensory memory. Nature, 372, 90-92. doi:10.1038/372090a0

Toiviainen, P., Tervaniemi, M., Louhivuori, J., Saher, M., Huotilainen, M., \& Näätänen, R. (1998). Timbre similarity: Convergence of neural, behavioral, and computational approaches. Music Perception, 16, 223-241.

Turpin, G., Schaefer, F., \& Boucsein, W. (1999). Effects of stimulus intensity, risetime, and duration on autonomic and behavioral responding: Implications for the differentiation of orienting, startle, and defense responses. Psychophysiology, 36(4), 453-463. doi: $10.1111 / 1469-8986.3640453$

Upham, F. (2014). Respiration rate script [Computer Software, Matlab]. Retrieved from https://github.com/finn42/RespirationTracking.

Van der Zwaag, M. D., Westerink, J. H. D. M., \& van den Broek, E. L. (2011). Emotional and psychophysiological responses to tempo, mode, and percussiveness. Musicae Scientiae, 15(2), 250-269. doi:10.1177/1029864911403364

Wessel, D., Bristow, D., \& Settel, Z. (1987) Control phrasing and articulation in synthesis. In Proceedings of the 1987 International Computer Music Conference, Champaign/Urbana, IL (pp. 108-116). San Francisco, CA: Computer Music Association. 